\title{
Focal Region Field of a Two Dimensional Gregorian System Coated with Isotropic Chiral Medium
}

\section{Tariq Rahim, Junaid Mughal, Mazhar Hussnain}

Faculty of Electronic Engineering, GIK Institute of Engineering Sciences and Technology, Topi, Swabi, N.W.F.P., Pakistan.

Email: rahim372@gmail.com, junaid@giki.edu.pk,mazhar_hussnain@yahoo.com

Received March $3^{\text {rd }}, 2010$; revised May 5 ${ }^{\text {th }}, 2010$; accepted May 10 ${ }^{\text {th }}, 2010$.

\begin{abstract}
Focal region field of a two dimensional Gregorian system coated with chiral medium is analyzed at high frequency. Maslov's method is used because the Geometrical Optics approximation fails at focal points. Maslov's method combines the simplicity of ray theory and the generality of Fourier transform. Fields patterns are calculated numerically and the results are plotted. The effects of thickness of chiral layer, chirality parameter of the chiral medium and permittivity of the medium are analyzed. The problem of simple dielectric layer is discussed as a special case of this problem.
\end{abstract}

Keywords: Chiral Medium, Maslov,Smethod, Gregorian System, Focal Region Field

\section{Introduction}

The knowledge of focal region field of focusing systems is useful for synthesizing feed arrays in imaging and design of multiple beam antennas in communication systems. The focusing of electromagnetic waves into material media is also a subject of considerable current interest due to applications in hyperthermia, microscopy, and optical data storage. Geometrical optics (GO) approximation is one of the well known method for evaluating high frequency field. It has been widely used to study various kinds of problems in different areas of electromagnetics, acoustics and seismology [1-3]. GO approximation for wave solution is important in electromagnetics because it provide insight into the behavior of wave front. GO is used only for high frequency approximation of a wave, provided the ray tube does not vanish. However, at caustic regions the ray tube shrinks to zero and GO show singularity at these regions. These regions are of great importance in all practical problems e.g. parabolic, paraboloidal and circular reflectors etc. To avoid these singularities Maslov proposed a method based on Maslov`s theory [4,5]. Maslov's method has been used to find the field at caustic regions [6-20]. The idea in Maslov's method provides a systematic procedure for predicting the field in the caustic region by combining the simplicity of ray theory and generality of the transform method. High frequency field expressions has been derived around feed point of a two dimensional Gregorian system using the Maslov's method in [19]. The same focussing system has been coated with isotropic and homogeneous chiral medium and field expressions are obtained. In Section 2 the plane wave reflection from a chiral slab backed by perfect electric conducting (PEC) plane is considered. In Section 3 high frequency expression for the field of a chiral coated Gregorian system excited by plane wave is derived. Numerical results and discussion are presented in Section 4 and the paper is concluded in Section 5.

\section{Reflection of Plane Waves From a Chiral Slab Backed by Conductor}

In this paper we want to find the reflected field around the focal region of a two dimensional Gregorian's main parabolic reflector coated with chiral medium. To achieve this the reflection of plane waves from a chiral slab backed by perfect electric conducting (PEC) plane is discussed as in $[15,21]$. As shown in Figure 1 the region $\mathrm{z} \leq 0$ is occupied by free space defined by following equations

$$
\begin{aligned}
& D=\varepsilon_{0} E \\
& B=\mu_{0} H
\end{aligned}
$$

and the region $0 \leq z \leq d$ is occupied by the chiral medium defined by Drude-Born-Fadorov (DBF) constitutive 
relations [21] as follows

$$
\begin{aligned}
& D=\varepsilon(E+\beta \nabla \times E) \\
& B=\mu(H+\beta \nabla \times H)
\end{aligned}
$$

The PEC is placed at $z=d$ as shown in Figure 1. The reflection coefficients for the parallel and perpendicular components of polarization is calculated in [11]

$$
\left[B_{\perp} B_{\|}\right]=\left([r]+[T]\left[[\Delta]\left[R_{3}\right][\Delta]-[R]\right)^{-1}[t]\right)\left[A_{\perp} A_{A}\right]
$$

In the above equation, $A_{\perp}, A$ and $B_{\perp}, B_{\Perp}$ are the parallel and perpendicular components of polarization of the incident and reflected fields respectively. $[r],[T],[R]$ and $[t]$ are $2 \times 2$ matrices. Elements of the matrices, which are Fresnel coefficients, are given in [15]. $\Delta$ and $R_{3}$ are also $2 \times 2$ matrices and are given in [15]. Using these reflection coefficients, the focal region fields of a two dimensional Gregorian's system are derived.

\section{Focal Region Field of Two Dimensional Gregorian Reflector}

Gregorian system consists of two reflectors, one is parabolic main reflector and another is hyperbolic sub-reflector as shown in Figure 2. This system has several advantages over a single parabolic reflector.In this paper we want to study the caustic region field of a two dimensional Gregorian system when the main reflector is coated with chiral medium using GO and Maslov's method. The equations of each reflector are given as follows

$$
\begin{aligned}
& \zeta_{1}=\frac{\xi_{1}^{2}}{4 f}-f+c, \zeta_{2}=a\left[\frac{1-\xi_{2}}{b^{2}}\right]^{\frac{1}{2}} c^{2}=a^{2}+b^{2} \\
& a R_{2}=c \zeta_{2}-a^{2}, \quad a R_{1}=c \zeta_{2}+a^{2}
\end{aligned}
$$

In the above relations $\left(\xi_{1}, \zeta_{1}\right)$ and $\left(\xi_{2}, \zeta_{2}\right)$ are the Cartesian coordinates of the point on the parabolic and elliptical reflectors, respectively. The Incident wave traveling along the negative $z$-axis is given by

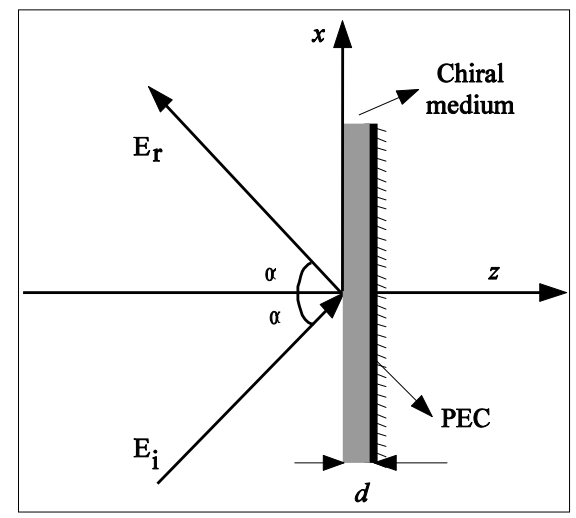

Figure 1. Reflection from a chiral slab backed by PEC

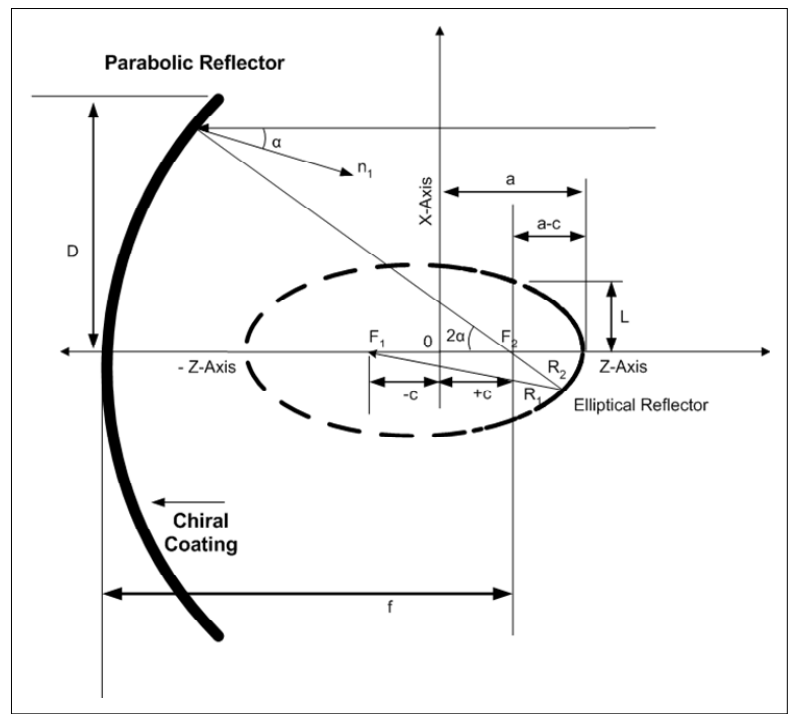

Figure 2. Gregorian antenna coated with chiral medium

$$
E^{i}=\left(A_{y} i_{y}+A_{x} i_{x}\right) \exp (j k z)
$$

where, $A_{y}, A_{x}$ are the components along $y$-axis and $x$-axis of the initial amplitude of the incident field respectively. The wave reflected from the hyperboloidal surface is given by

$$
\begin{aligned}
E^{r}= & {\left[B_{\perp} i_{y}+B_{1}\left\{-\sin (2 \alpha-2 \psi) i_{x}-\cos (2 \alpha-2 \psi) i_{i}\right\}\right] } \\
& \times \exp \left[-j k z\left\{\sin (2 \alpha-2 \psi) i_{x}+\cos (2 \alpha-2 \psi) i_{z}\right\}\right]
\end{aligned}
$$

The wave vector of the wave reflected by the parabolic reflector is given by

$$
p_{1}^{r}=-\sin 2 \alpha i_{x}+\cos 2 \alpha i_{r}
$$

and the wave vector of the wave reflected by the hyperbolic reflector is

$$
p_{2}^{r}=-\sin (2 \alpha-2 \psi) i_{x}-\cos (2 \alpha-2 \psi) i_{2}
$$

where, the angle $\alpha$ and angle $\psi$ are given by the relation

$$
\begin{aligned}
\sin \alpha & =\frac{\xi_{1}}{\sqrt{\xi_{1}^{2}+4 f^{2}}}, \cos \alpha=\frac{2 f}{\sqrt{\xi_{1}^{2}+4 f^{2}}} \\
\sin \psi=-\frac{1}{\sqrt{R_{1} R_{2}}} \frac{a}{b} \xi_{2}, \cos \psi & =\frac{1}{\sqrt{R_{1} R_{2}}} \frac{b}{a} \zeta_{2}
\end{aligned}
$$

In the above equations $R_{1}$ and $R_{2}$ are the distances from the point $\left(\xi_{2}, \zeta_{2}\right)$ to the focal points $z=-c$ and $z=c$, respectively. The unit normal vectors to the parabolic and hyperbolic surfaces are given by $n_{1}$ and $n_{2}$ respectively. These normals can be written as

$$
n_{1}=-\sin \alpha i_{x}+\cos \alpha i_{2}, n_{2}=-\sin \psi i_{x}+\cos \psi i_{2}
$$


The general form of the geometrical optics solution for the wave is given by [19]

$$
E(r)=E_{0}\left\{\frac{D(\tau)}{D(0)}\right\}^{\frac{-1}{2}} \exp \left\{-j k_{0}\left(S_{0}+\tau\right)\right.
$$

The Jacobian associated with the wave reflected by the parabolic reflector is given by [19]

$$
J(\tau)=\frac{D(\tau)}{D(0)}=1-\frac{\cos ^{2} \alpha}{f} \tau
$$

The Cartesian coordinates of the ray reflected by the hyperbolic surface is given by

$$
\begin{aligned}
& x=\xi_{2}+p_{x 2} \tau=\xi_{1}+p_{x 1} \tau_{1}+p_{x 2} \tau \\
& z=\zeta_{2}+p_{22} \tau=\zeta_{1}+p_{x 1} \tau_{1}+p_{22} \tau
\end{aligned}
$$

where, $\tau_{1}=\sqrt{\left(\xi_{1}-\xi_{2}\right)^{2}+\left(\zeta_{1}-\zeta_{2}\right)^{2}}$. In the above equation $\left(p_{x 1}, p_{21}\right)$ and $\left(p_{x 2}, p_{z_{2}}\right)$ are the rectangular components of reflected wave vectors $p_{r}^{1}$ and $p_{r}^{2}$, respectively. Now we consider the field after the reflection from the hyperbolic cylinder. The transformation from the Cartesian coordinates $(x, z)$ to the ray coordinates $\left(\xi_{1}, \tau\right)$ is given by [19]

$$
D(\tau)=\frac{\cos ^{2} \alpha}{f} R_{2}\left[1-\frac{\tau}{R_{1}}\right]
$$

Thus the geometrical ray expression of each component of the reflected wave is

$$
\begin{aligned}
& E_{x}^{r}=-\cos (2 \alpha-2 \psi) B_{\|}\left[1-\frac{\tau}{R_{1}}\right]^{-\frac{1}{2}} \exp \left[-j k\left(S_{0}+\tau_{1}+\tau\right)\right] \\
& E_{y}^{r}=B_{\perp}\left[1-\frac{\tau}{R_{1}}\right]^{-\frac{1}{2}} \exp \left[-j k\left(S_{0}+\tau_{1}+\tau\right)\right] \\
& E_{z}^{r}=-\sin (2 \alpha-2 \psi) B_{\|}\left[1-\frac{\tau}{R_{1}}\right]^{-\frac{1}{2}} \exp \left[-j k\left(S_{0}+\tau_{1}+\tau\right)\right]
\end{aligned}
$$

where, $E_{0}^{r}$ is the amplitude of the incident wave at the reflection point on the parabolic surface and

$$
\begin{aligned}
& S_{0}=-\zeta_{1}=2 f \frac{\cos 2 \alpha}{1+\cos 2 \alpha}-c \\
& \tau_{1}=\sqrt{\left(\xi_{2}-\xi_{1}\right)^{2}+\left(\zeta_{2}-\zeta_{1}\right)^{2}} \\
& \tau=\sqrt{\left(x-\xi_{2}\right)^{2}+\left(z-\zeta_{2}\right)^{2}}
\end{aligned}
$$

As indicated by (7), $E(r)$ will become infinite for
$\frac{D(\tau)}{D(0)}=0$ i.e. at the caustic points. The expression which is valid at the focal point according to Maslov's method is given by [19]

$$
\begin{aligned}
E(r)= & \sqrt{\frac{k_{0}}{j 2 \pi}} \int_{-\infty}^{\infty} A_{0}(\xi)\left[J(\tau) \frac{\partial p_{z}}{\partial z}\right] \\
& \times \exp \left\{-j k_{0}\left[S_{0}+\tau-\left(x, p_{z}\right) p_{z}+z p_{z}\right]\right\}
\end{aligned}
$$

The amplitude term $\left[J(\tau) \frac{\partial p_{z 2}}{\partial z}\right]^{-\frac{1}{2}}$ in the above equation is given by [19]

$$
\left[J(\tau) \frac{\partial p_{z 2}}{\partial z}\right]^{-\frac{1}{2}}=\frac{\sqrt{R_{1}}}{\sin (2 \alpha-2 \psi)}
$$

The phase function is given by

$$
S=S_{0}+\tau_{1}+\tau-z\left(x, p_{z_{2}}\right) p_{z_{2}}+p_{z_{2}} z
$$

where, $S_{0}+\tau_{1}$ is given by (15). The extra term is given by

$$
\begin{aligned}
S_{e x}= & \tau-z\left(x, p_{z_{2}}\right) p_{z_{2}}+p_{z_{2}} z \\
= & \tau-\left(\zeta_{2}+\mathrm{p}_{z_{2}} \tau\right) \mathrm{p}_{z_{2}}+\mathrm{p}_{z_{2}} \\
= & \left(\mathrm{p}_{x_{2}}\right)^{2} \tau+\left(\mathrm{z}-\zeta_{2}\right) \mathrm{p}_{z_{2}} \\
= & \mathrm{p}_{x_{2}}\left(\mathrm{x}-\xi_{2}\right)+\left(\mathrm{z}-\zeta_{2}\right) \mathrm{p}_{z_{2}} \\
= & -\rho \cos (2 \alpha-2 \psi-\phi) \\
& +\left[\sin (2 \alpha-2 \psi) \xi_{2}+\cos (2 \alpha-2 \psi) \zeta_{2}\right]
\end{aligned}
$$

We substitute (19)-(20) into (18) and taking $A_{0}(\xi)=1$, we can find the finite field around the caustic as given below

$$
\begin{aligned}
E_{x}^{r}= & \sqrt{\frac{k}{j 2 \pi}}\left[\int_{A_{1}}^{A_{2}}+\int_{-A_{2}}^{A_{1}}\right] \cos (2 \alpha-2 \psi) B_{\|} \sqrt{R_{1}} \\
& \times \exp \left[-j k\left(S_{0}+\tau_{1}+S_{e x}\right)\right] d(2 \alpha) \\
E_{y}^{r}= & \sqrt{\frac{k}{j 2 \pi}\left[\int_{A_{1}}^{A_{2}}+\int_{A_{2}}^{A_{1}}\right] B_{\perp} \sqrt{R_{1}}} \\
& \times \exp \left[-j k\left(S_{0}+\tau_{1}+S_{e x}\right)\right] d(2 \alpha) \\
E_{z}^{r}= & \sqrt{\frac{k}{j 2 \pi}}\left[\int_{A_{1}}^{A_{2}}+\int_{-A_{2}}^{A_{1}}\right] \sin (2 \alpha-2 \psi) B_{\|} \sqrt{R_{1}} \\
& \times \exp \left[-j k\left(S_{0}+\tau_{1}+S_{e x}\right)\right] d(2 \alpha)
\end{aligned}
$$

In the above equation, $R_{1}, S_{0}, \tau_{1}$ and $S_{e x}$ are expressed in terms of $\alpha$ and $A_{1}$ and $A_{2}$ are the subtention angles $2 \alpha$ at the edges of the parabolic and hyperbolic surfaces. The limit of integration are calculated by the expression 


$$
A_{1}=2 \arctan \left(\frac{D}{2 f}\right), A_{2}=\arctan \left(\frac{L}{2 c}\right)
$$

\section{Result and Discussion}

Field pattern around the caustic region of a Gregorian system are determined using (21-23) by performing the integration numerically. The line plots of the field around the focal region located between the two reflectors, that point $\mathrm{F}_{2}$ in Figure 2. Simulation were done for $A_{1}=$ $\pi / 4.1, \quad A_{2}=\pi / 6.5, \quad k_{0}=1, \quad f=100, \quad \mu=\mu_{0}$ and different values of $\varepsilon=\varepsilon / \varepsilon_{0}, d$, and $\beta$. We have considered two types of polarization for incident wave. One is $\left(A_{x}=0, A_{y}=1\right)$ and the other is $\left(A_{x}=1\right.$, $\left.A_{y}=0\right)$. The results are plotted in Figures 3-20. In all figures horizontal axis is $\mathrm{z}$-axis and vertical axis corresponds to the absolute value of reflected field component. All plots are taken at $x=0$. We have studied the effects of thickness of the the coated layer $(d)$, the chirality parameter $(\beta)$ and the relative permittivity of the me-

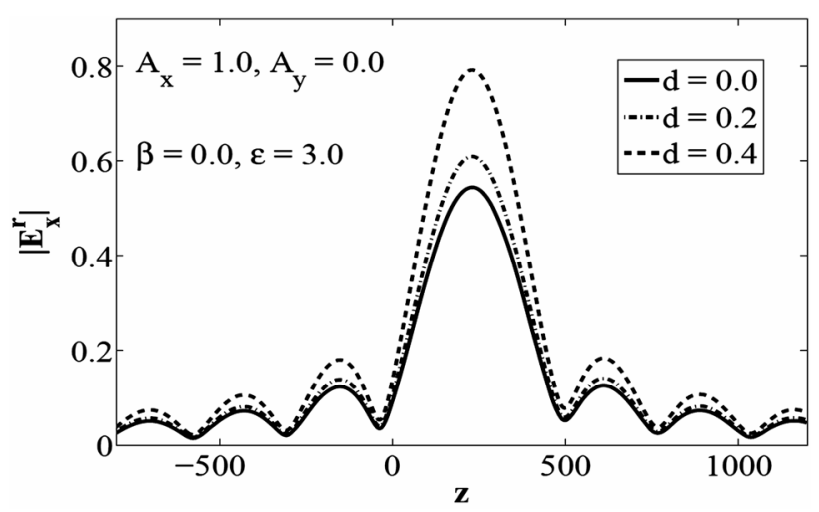

Figure 3. Plot of $\left|E_{x}^{r}\right|$, when parabolic reflector is coated with dielectric layer of varying thickness $d$

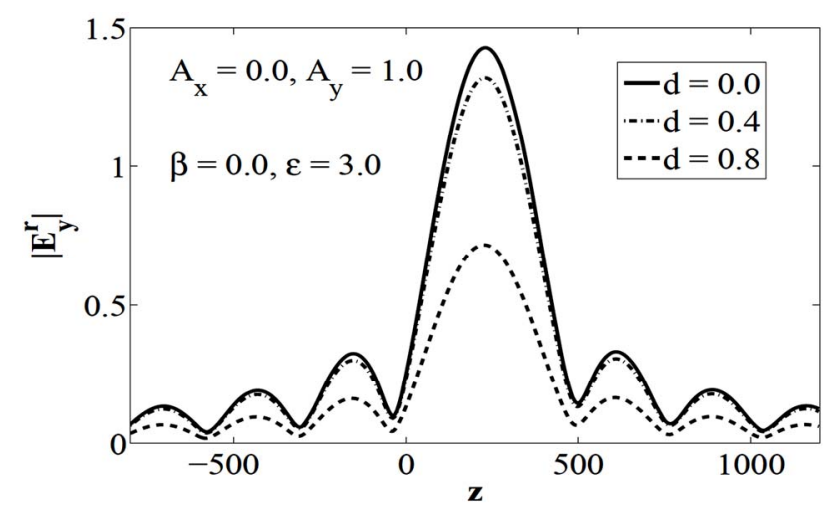

Figure 4. Plot of $\left|E_{y}^{r}\right|$, when parabolic reflector is coated with dielectric layer of varying thickness $d$ dium $(\varepsilon$ ) on the focal region field. Figures 3-5 show the effect of increase in the value of $d$ keeping $\beta=0$ and $\varepsilon=3$, that is ordinary dielectric case. We have shown in Figure 3 and 5the $\left|E_{x}^{r}\right|$ and $\left|E_{z}^{r}\right|$ for $\left(A_{x}=1, A_{y}=0\right)$. These figures show that increase in thickness of the coated layer $(d)$ results an increase in $\left|E_{x}^{r}\right|$ and $\left|E_{z}^{r}\right|$. Figure 4 shows the plot of $\left|E_{y}^{r}\right|$ for $\left(A_{x}=0, A_{y}=1\right)$. This figure shows decrease in field strength as $d$ increases. Figures 6-11 show the effect of $d$ while keeping $\beta=0.5$ and $\varepsilon=1$ for both types of polarization. Figures 7 and $\mathbf{1 1}$ show increase in the field strength of $\left|E_{x}^{r}\right|$ and $\left|E_{z}^{r}\right|$ for $\left(A_{x}=1, A_{y}=0\right)$, respectively. Figure 6 and $\mathbf{1 0}$ show the cross polarization effect due to chiral medium for polarization $\left(A_{x}=0, A_{y}=1\right)$, which also increases with increase in $d$. For $d=0$, cross polarization effect vanishes and field strength increases with increase in $d$. Figure 8 shows the plot of $\left|E_{y}^{r}\right|$ for $\left(A_{x}=0, A_{y}=1\right)$, which decreases with increase in $d$, and Figure 9

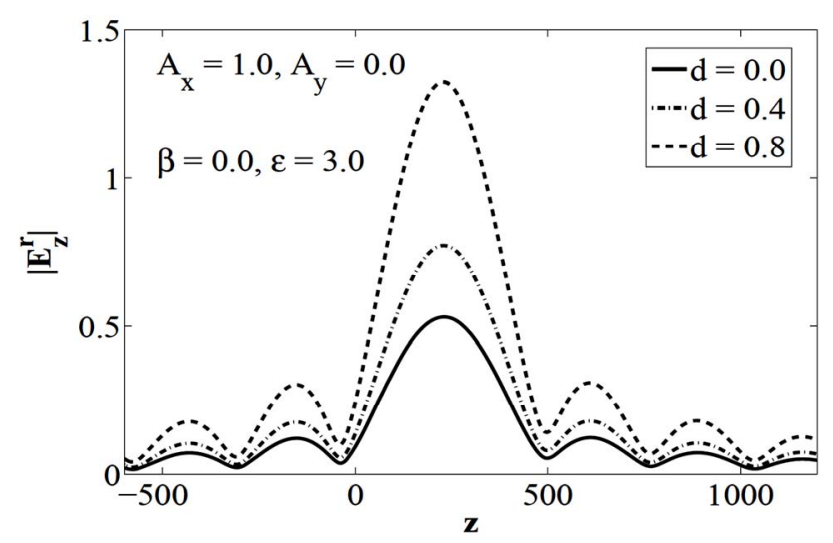

Figure 5. Plot of $\left|E_{z}^{r}\right|$, when parabolic reflector is coated with dielectric layer of varying thickness $d$

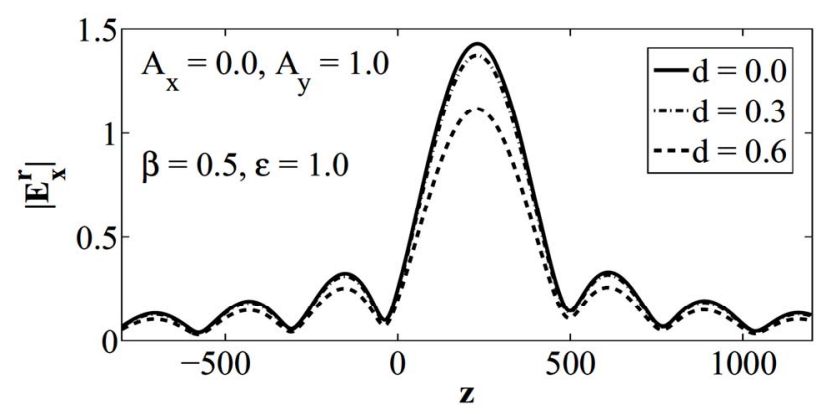

Figure 6. Plot of $\left|E_{x}^{r}\right|$, when the thickness of the coated layer $d$ is varying. The impedance of chiral medium is equal to that of free space 


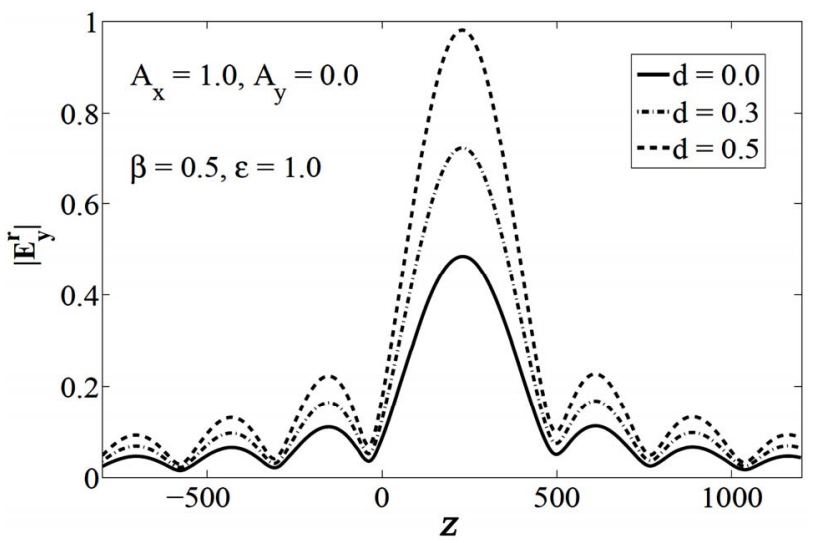

Figure 7. Plot of $\left|E_{x}^{r}\right|$, when the thickness of the coated layer $\mathrm{d}$ is varying. The impedance of chiral medium is equal to that of free space

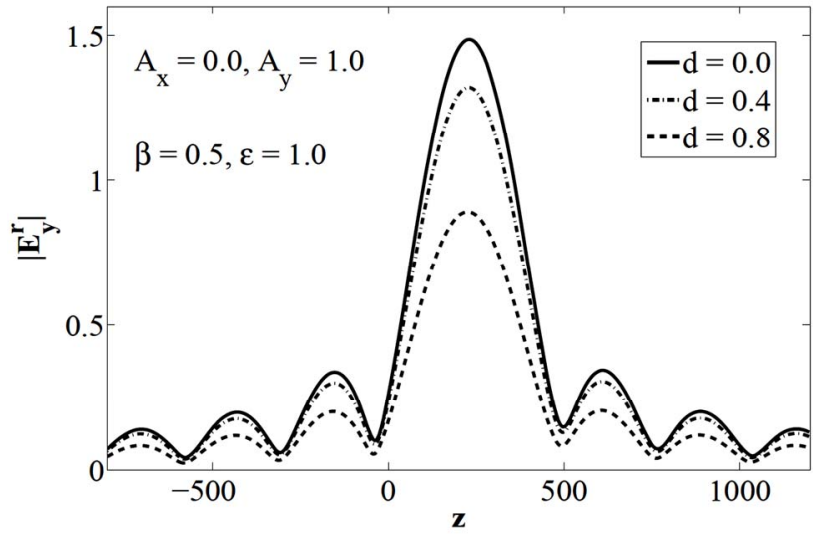

Figure 8. Plot of $\left|E_{y}^{r}\right|$, when the thickness of the coated layer $\mathbf{d}$ is varying. The impedance of chiral medium is equal to that of free space

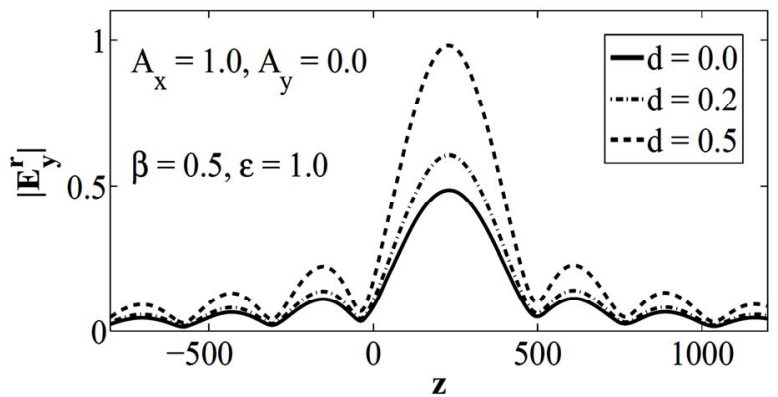

Figure 9. Plot of $\left|E_{y}^{r}\right|$, when the thickness of the coated layer $d$ is varying. The impedance of chiral medium is equal to that of free space

shows the plot for $\left(A_{x}=1, A_{y}=0\right)$. This figure shows that cross polarization effect are zero for $d=0$, but increases with increase in layer thickness $d$.

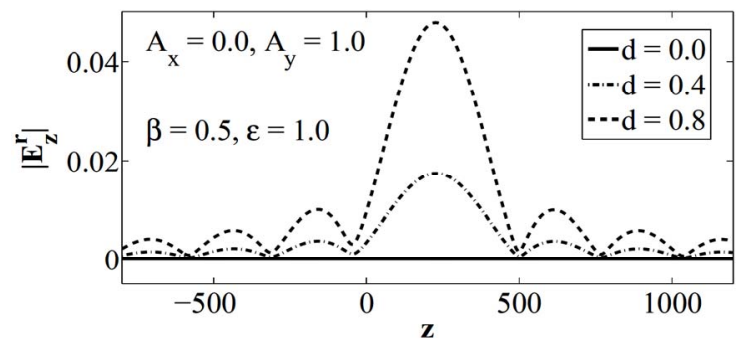

Figure 10. Plot of $\left|E_{z}^{r}\right|$, when the thickness of the coated layer $\mathrm{d}$ is varying. The impedance of chiral medium is equal to that of free space

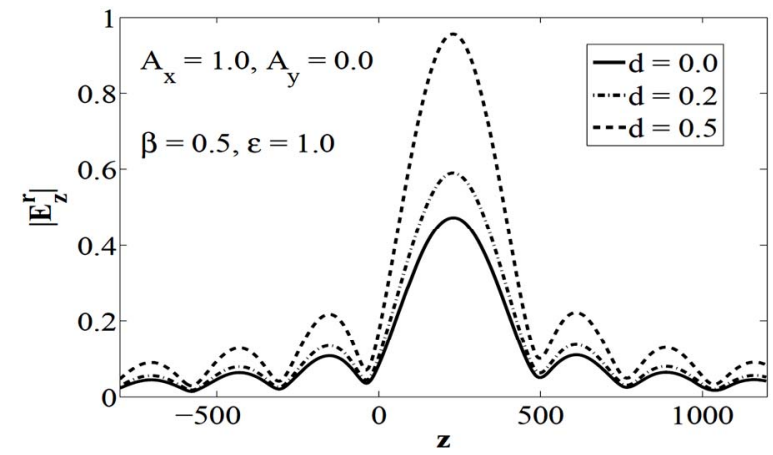

Figure 11. Plot of $\left|E_{z}^{r}\right|$, when the thickness of the coated layer $d$ is varying. The impedance of chiral medium is equal to that of free space

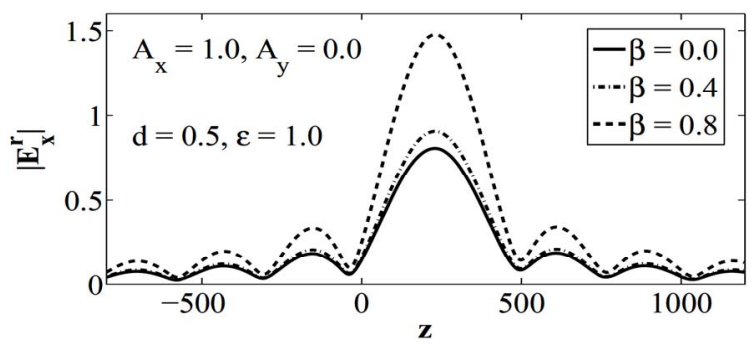

Figure 12. Plot of $\left|E_{x}^{r}\right|$ showing the effect of chirality parameter $\beta$. The impedance of chiral medium is equal to that of free space

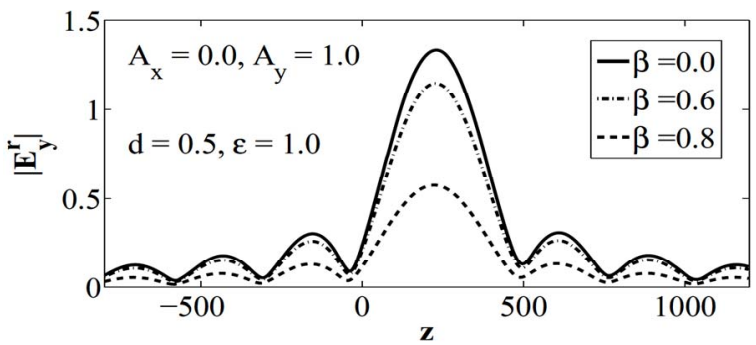

Figure 13. Plot of $\left|E_{y}^{r}\right|$ showing the effect of chirality parameter $\beta$. The impedance of chiral medium is equal to that of free space 


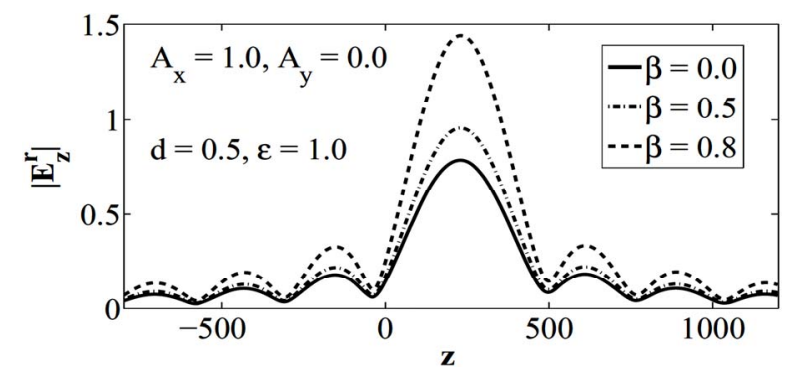

Figure 14. Plot of $\left|E_{z}^{r}\right|$ showing the effect of chirality parameter $\beta$. The impedance of chiral medium is equal to that of free space

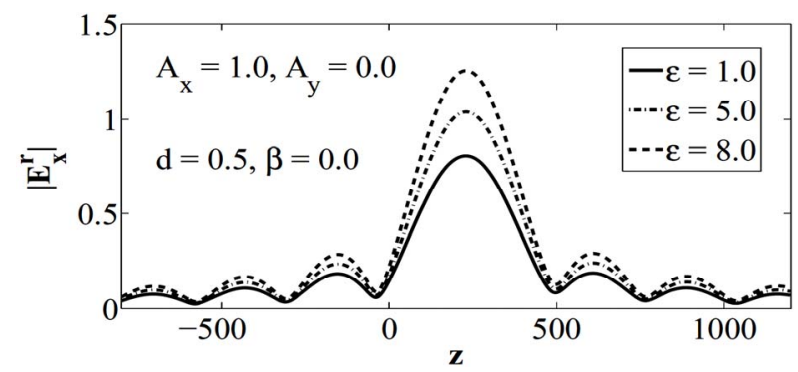

Figure 15. Plot of $\left|E_{x}^{r}\right|$ showing the effect of relative permittivity $\varepsilon$ of dielectric layer

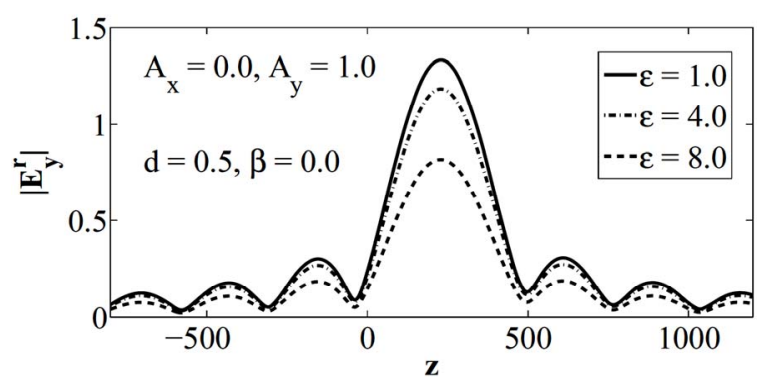

Figure 16. Plot of $\left|E_{y}^{r}\right|$ showing the effect of relative permittivity $\varepsilon$ of dielectric layer

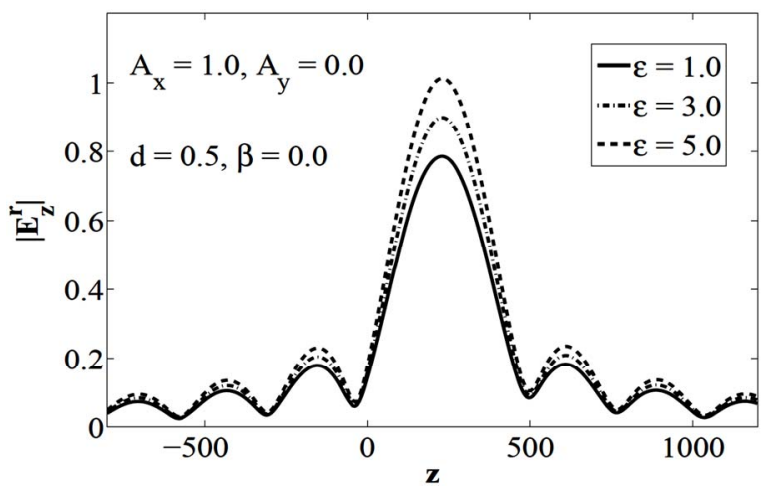

Figure 17. Plot of $\left|E_{z}^{r}\right|$ showing the effect of relative permittivity $\varepsilon$ of dielectric layer

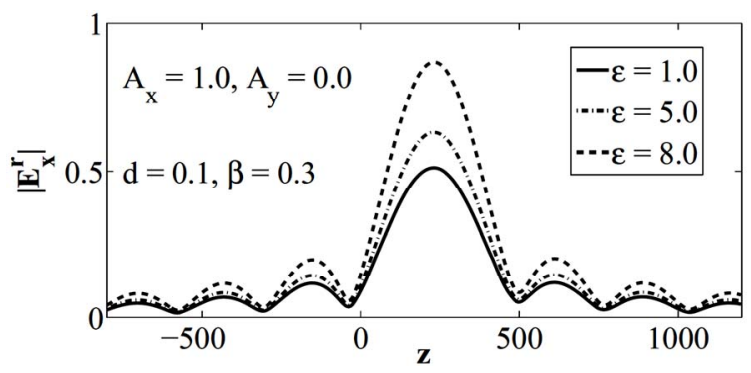

Figure 18. Plot of $\left|E_{x}^{r}\right|$ showing the effect of relative permittivity $\varepsilon$ of chiral layer

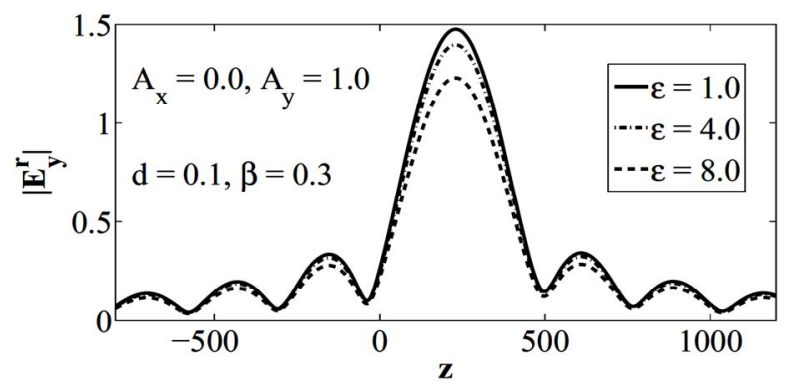

Figure 19. Plot of $\left|E_{y}^{r}\right|$ showing the effect of relative permittivity $\varepsilon$ of chiral layer

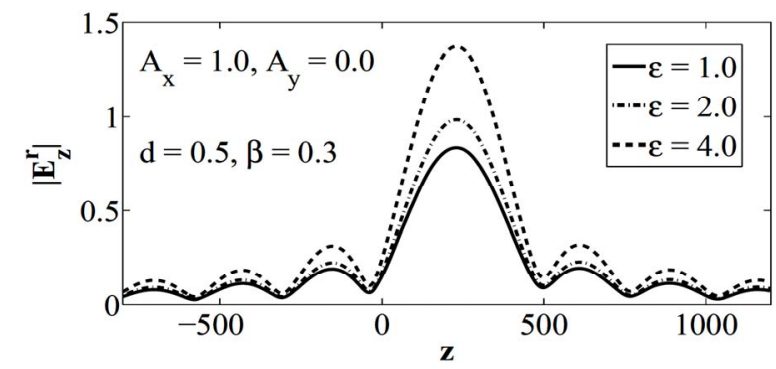

Figure 20. Plot of $\left|E_{z}^{r}\right|$ showing the effect of relative permittivity $\varepsilon$ of chiral layer

Figures 12 and 14 show the effect of chirality parameter $\beta$ on field components while keeping $\varepsilon=1$ and $d=0.5$. Figures 12 and 14 show the plots of $\left|E_{x}^{r}\right|$ and $\left|E_{z}^{r}\right|$ for $\left(A_{x}=1, A_{y}=0\right)$ respectively. These figures show that field strength increases with increase in chirality parameter $\beta$. Figure 13 shows that $\left|E_{y}^{r}\right|$ decreases with increase in $\beta$. Cross polarization effects have not been shown because they have same trends as discussed above. Figures 15-17 show the effect of $\varepsilon$ while keeping $d=0.5$ and $\beta=0$, that is for ordinary dielectric case. Figures 15 and 17 show the plot of $\left|E_{x}^{r}\right|$ and $\left|E_{z}^{r}\right|$ for polarization $\left(A_{x}=1, A_{y}=0\right)$ respectively and show the increase in the field strength if 
we increase the relative permittivity of dielectric medium coated on the surface of reflector. Figure 16 shows that $\left|E_{y}^{r}\right|$ decreases with increase in $\varepsilon$ for polarization $\left(A_{x}=0, A_{y}=1\right)$. Figures 18-20, which are for chiral coating, show the same trends as in Figures 15-17.

\section{Conclusions}

Focal region fields of a two dimensional Gregorian reflector is analyzed using GO and Maslov's method. Two types of polarization are discussed. The reflected field components are analyzed numerically and the results are given in the focal plane. It is concluded that increase in chirality parameter $(\beta)$, increase in thickness $(d)$ and relative permittivity $(\varepsilon)$, results in increase in of $\left|E_{x}^{r}\right|$ and $\left|E_{z}^{r}\right|$ for $\left(A_{x}=1, A_{y}=0\right)$. While $\left|E_{y}^{r}\right|$ decreases for $\left(A_{x}=0, A_{y}=1\right)$. Cross polarized fields exist when $k \beta \neq 0$ and increase with increase in value of $d$.

\section{REFERENCES}

[1] Y. A. Kravtsov and Y. I. Orlov, "Caustics, Catastrophes, and Wave Fields,” Springer Verlag, Berlin, 1993.

[2] G. A. Dechamps, "Ray Techniques in Electromagnetics," Proceedings of IEEE, 1972, pp. 1022-1035.

[3] C. H. Chapman and R. Drummond, "Body Wave Seismograms in Inhomogeneous Media Using Maslov Asymptotic Theory," Bulletin of the Seismological Society of America, Vol. 72, No. 68, 1982, pp. 277-317.

[4] V. P. Maslov, "Perturbation Theory and Asymptotic Methods," Moskov., Gos., University, Moscow, 1965 (in Russian). (Translated into Japanese by Ouchi et al., Iwanami).

[5] V. P. Maslov and V. E. Nazaikinski, "Asymptotic of Operator and Pseudo-Differential Equations,” Consultants Bureau, New York, 1988.

[6] A. Ghaffar, Q. A. Naqvi and K. Hongo, "Analysis of the Fields in Three Dimensional Cassegrain System," Progress in Electromagnetics Research, Vol. 72, 2007, pp. 215-240.

[7] A. Hussain, Q. A. Naqvi and K. Hongo, "Radiation ChaRacteristics of the Wood Lens Using Maslov's Method," Progress in Electromagnetics Research, Vol. 73, 2007, pp. 107-129.

[8] Y. Ji and K. Hongo, "Analysis of Electromagnetic Waves Refracted by a Spherical Dielectric Interface Method,” Journal of the Optical Society of America, Vol. 8, No. 3, 1991, pp. 541-548.

[9] Y. Ji and K. Hongo, "Field in the Focal Region of a Dielec-Tric Spherical by Maslov's Method,” Optical Society of America. Vol. 8, No. 11, 1991. pp. 1721-1728.

[10] K. Hongo, Y. Ji and E. Nakajima, "High Frequency Ex- pression for the Field in the Caustic Region of a Reflector Using Maslov's Method,” Radio Science, Vol. 21, No. 6, 1986, pp. 911- 919.

[11] K. Hongo and Y. Ji, "High Frequency Expression for the Field in the Caustic Region of a Cylindrical Reflector Using Maslov’s Method,” Radio Science, Vol. 22, No. 3, 1987, pp. 357-366,

[12] K. Hongo and Y. Ji, "Study of the Field Around the Focal Region of Spherical Reflector Antenna by Maslov's MetHod,” IEEE Transaction Antennas Propagat, Vol. 36, No. 5, 1988, pp. 592-598.

[13] R. W. Ziolkowski and G. A. Deschamps, “Asymptotic Evaluation of High Frequency Field near a Caustic: An Intr- Oduction to Maslov's Method,” Radio Science, Vol. 19, No. 4, 1984, pp. 1001-1025.

[14] M. Faryad and Q. A. Naqvi, "High Frequency Expression for the Field in the Caustic Region of Cylindrical Reflector Placed in Chiral Medium," Progress in Electromagnetics Research, Vol. 76, 2007, pp. 153-182.

[15] Faryad, M. and Q. A Naqvi, "High Frequency Expression for the Field in the Caustic Region of a Parabolic Reflector Coated with Isotropic Chiral Medium," Journal of Electromagnetic Waves and Application, Vol. 22, 2008, pp. 965-986.

[16] M. Faryad and Q. A. Naqvi, "Cylindrical Reflector in Chiral Medium Supporting Simultaneously Positive Phase Velocity and Negative Phase Velocity,” Journal of Electromagnetic Waves and Applications, Vol. 22, No. 2, 2008, pp. 563-572.

[17] A. Ghaffar, A. Hussain, Q. A. Naqvi and K. Hongo, "Radiation Characteristics of an Inhomogeneous Slab Using Maslov's Method," Journal of Electromagnetic Waves and Applications, Vol. 22, No. 2, 2008, pp. 301-312.

[18] A. Aziz, A. Ghaffar, Q. A. Naqvi and K. Hongo, "Analysis of the Fields in Two Dimensional Gregorian System," Journal of Electromagnetic Waves and Applications, Vol. 22, No. 1, 2008, pp. 85-97.

[19] A. Aziz, Q. A. Naqvi and K. Hongo, "Analysis of the Fields in Two Dimensional Cassegrain System,” Progress in Electromagnetics Research, Vol. 71, 2007, pp. 227-241.

[20] A. Ghaffar, A. Rizvi and Q. A. Naqvi, "Field in the Focal Space of Symmetrical Hyperboloidal Focusing Lens," Progress in Electromagnetics Research, Vol. 89, 2009, pp. 255-273.

[21] A. Lakhtakia, "Beltrami Fields in Chiral Media,” World Scientific Series in Contemporary Chemical Physics, 1994.

[22] S. Bassiri, "Electromagnetic Waves in Chiral Media," Recent Advances in Electromagnetic Theory, H. N. Kritikos and D. L. Jaggard, Eds., Springer-Verlag, New York, 1990.

[23] V. Tuz and V. Kazanskiy, "Periodicity Defect Influence on the Electromagnetic Properties of a Sequence with Bi-Isotropic Layers," Progress in Electromagnetics Research B, Vol. 7, 2008, pp. 299-307. 